\title{
Velocity measurements of the liquid - gas flow using gamma absorption and modified conditional averaging
}

\author{
Robert Hanus ${ }^{1, a}$, Marcin Zych ${ }^{2}$, Adam Kowalczyk ${ }^{1}$ and Leszek Petryka ${ }^{2}$ \\ ${ }^{1}$ Rzeszow University of Technology, Rzeszow 35-959, Poland \\ ${ }^{2}$ AGH University of Science and Technology, Krakow 30-059, Poland
}

\begin{abstract}
The paper presents idea and an exemplary application of gamma-absorption in the measurement of gas bubbles transportation in a gas-liquid mixture flow through a horizontal pipeline. In the tests on laboratory installation two ${ }^{241} \mathrm{Am}$ radioactive sources and probes with $\mathrm{NaI}(\mathrm{Tl})$ scintillation crystals have been used. For analysis of electrical signals obtained from detectors the modified conditional averaging of the absolute value of delayed signal (CAAV) is proposed. The proposed method is based on the quotient of classical crosscorrelation (CCF) and CAAV. Results of the time delay estimation and gas-phase velocity measurements are compared with one obtained using CCF. The combined uncertainties of the mean velocity of air bubbles evaluation in the presented experiment did not exceed $2.1 \%$ (CCF) and $1.7 \%$ (CCF/CAAV), which is a satisfactory result in industrial applications.
\end{abstract}

\section{Introduction}

The flow when gas is transporting by liquid commonly occurs in industry, e.f. in nuclear, chemical and petrochemical engineering. To describe such flow it is required to record variation of the velocity or flow rate of each components, as well as their mixing and distribution between the transported streams. Direct measuring of these values is difficult, and requires the use of advanced techniques, employing computer tomography (resistive, capacitive, optical and X-rays), Coriolis flowmeters, optical equipment, and nuclear performances [1 - 9].

The routine mentioned lastly uses tracers injected under certain conditions into the flow (radiotracer method), or is based on the use of sealed radioactive sources (absorption method) [5 - 9]. Often in both cases scintillation probes are mounted outside the analysed stream as detectors of the radiation. The advantage of such methods is the simplicity and relatively high accuracy of provided information. The absorption measurement is non-invasive, and at the same time allows determining the velocity and concentration of a minority phase with use of the same equipment.

Frequently in such measurements the mutually delayed stochastic signals are provided by probes situated on walls of the pipeline or open channel. The time delay of these signals is used to determine the velocity of the minority phase and other flow parameters. The processing of measured signals usually is disturbed by noise, so requires the use of signal conditioning and statistical processing in the time or frequency domain.
The classical methods of the time delay estimation applied for stationary random signals include the crosscorrelation function (CCF) and the phase of crossspectral density [9-16]. Less popular method includes, among others, correlation analysis with the Hilbert Transform [13, 17, 18], differential methods [19 - 21] and methods based on the conditional averaging of the signals [22 - 25].

The paper consists of five sections. In the next one a basic of application of the gamma-absorption for twophase flow evaluation and short description of the laboratory installation are presented. The section 3 presents methods of the signal analysis: CCF, conditional average value of the absolute value of delayed signal (CAAV) and proposed combined $\mathrm{CCF} / \mathrm{CAAV}$ function. In this section the principle of calculation of the velocity of air bubbles in the gas-liquid mixture flow through horizontal pipeline is also described. In the next section exemplary results of measurements of the time delay and mean velocity of gaseous phase transported by liquid and their uncertainties are presented. The last section contains the summary of the presented study and final conclusions.

\section{Gamma-absorption in two-phase flow evaluation and laboratory installation}

The gamma absorption is based on exponential decreasing of a monoenergetic gamma beam in function of composition and geometry of absorbent:

\footnotetext{
$\overline{{ }^{a} \text { Corresponding author: rohan@prz.edu.pl }}$
} 


$$
J=J_{0} \exp (-\eta \cdot \mu \cdot x)
$$

where $J_{0}$ is the inlet to absorbent radiation intensity, $J$ is the outlet intensity detected after the beam has traveled a distance $x$ through the absorbing materials, $\eta$ and $\mu$ represents consequently the density and mass absorption coefficient of these materials [7].

If the basic equation is applied to an air-water mixture flow then the corresponding expression is:

$$
J=J_{0} \exp \left[-\left(\eta_{A} \cdot \mu_{A} \cdot x_{A}+\eta_{W} \cdot \mu_{W} \cdot x_{W}\right)\right],
$$

where indexes $A$ and $W$ for $\eta, \mu$ and $x$ denote the air and the water respectively.

The changes of the intensity of radiation are recorded by the scintillation detectors and converted into output electrical impulses [6].

The gamma-absorption measuring stand is presented in figure 1. Two sealed radioactive sources (1) emit gamma radiation beams shaped by collimators (2). Photons pass through the pipeline with analyzed air-water mixture (5) and collimator (3) before achieved the detector (4). In the presented experiment two sets consisting of linear ${ }^{241} \mathrm{Am}$ sources with an activity of $100 \mathrm{mCi}$ and probes with $\mathrm{NaI}(\mathrm{Tl})$ scintillation detectors are located with the distance of $L=97 \mathrm{~mm}$ between them. At the outputs of scintillation probes count rates $I_{x}(t)$ and $I_{y}(t)$ are recorded and processed after removal a background contribution. These values depend on the local condition of the flowing medium.

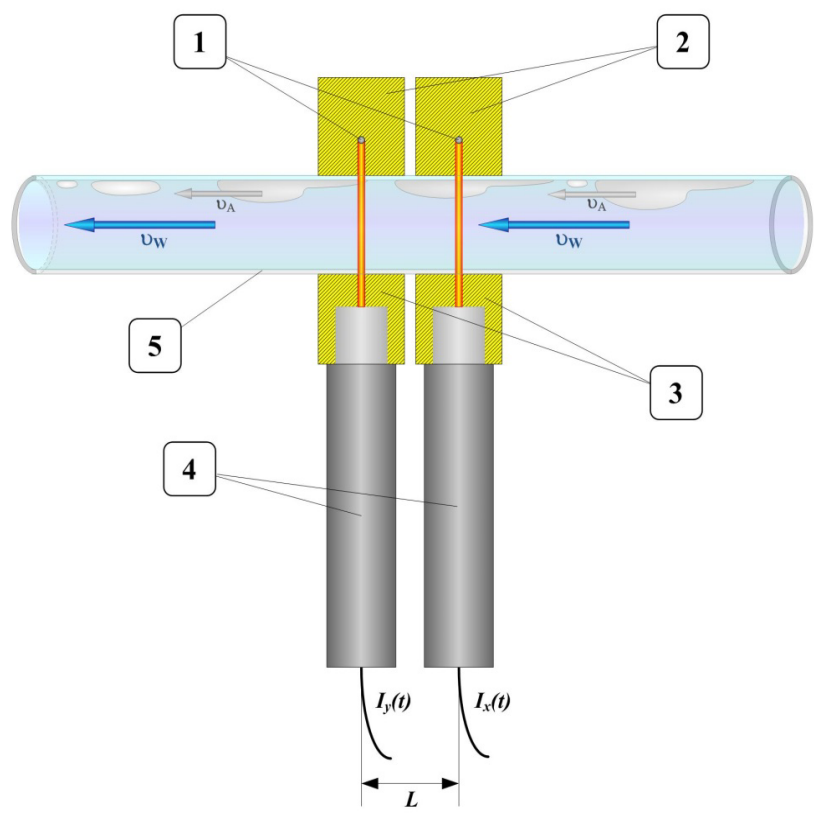

Figure 1. The $\gamma$-ray absorption stand: 1 - gamma radiation source, 2 - collimator of source, 3 - colli-mator of detector, 4 - scintillation probe, 5 - pipeline, $v_{A}, v_{W}$ - velocities of air and water respectively.

The measuring equipment presented in figure 1 was applied in the Industrial Radiometry Laboratory of the AGH University of Science and Technology in Krakow, Poland in an experimental hydraulic installation show in figure 2 .

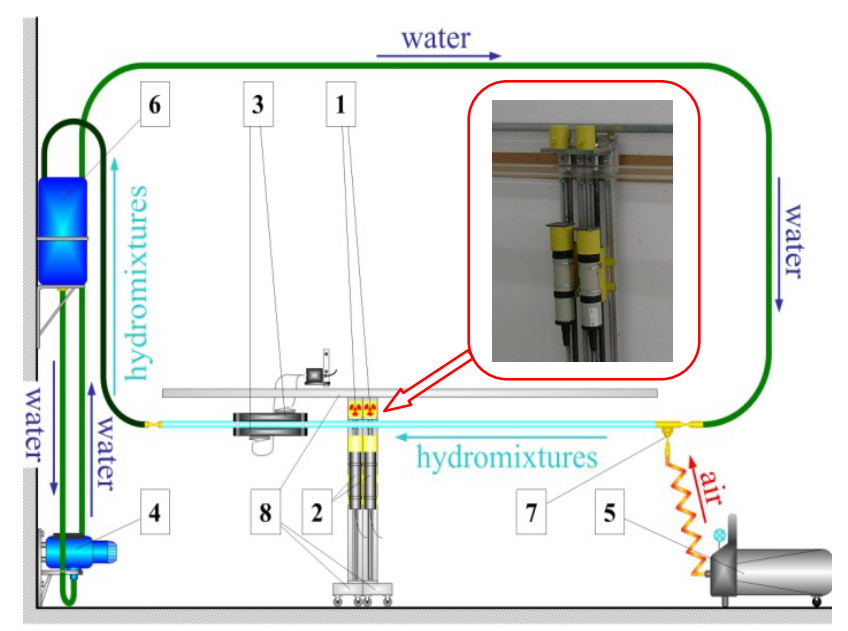

Figure 2. Scheme of experimental hydraulic installation: 1 - $\gamma$-ray sealed source, 2 - scintillation probe, 3 - ultrasonic flow meter, 4 - pump, 5 - air compressor, 6 - air-removing vessel, 7 - air nozzle, 8 - shifting system.

The installation consists of a transparent acrylic glass tube with internal diameter $30 \mathrm{~mm}$ and a length of $4.5 \mathrm{~m}$, to which water is pumped by a rotary pump (4) and air from the compressor (5) by an injector nozzle (7). Due to that the controlled mixture of water and air supply the measuring pipe and flowing up to the air-removing tank (6). The measurement of air bubbles velocity was arrange by the measuring system consisting of two $\gamma$-radioactive sources (1) and two scintillation probes (2). The measuring set is mounted on a special trolley (8), which allows moving the set along the pipeline. Independently water flow rate was continuously measured by Uniflow 990 ultrasonic flowmeter (3). The rotary pump (4) controlled by a waver allows selection of water velocity in the measuring pipe between $0.5-2.5 \mathrm{~m} / \mathrm{s}$.

The data acquisition equipment is comprised of PC with the dedicated counters card connected to a USB port. The voltage pulses $I_{x}(t)$ and $I_{y}(t)$ obtained from the detectors counted within the sampling time $\Delta t=1 \mathrm{~ms}$ create mutual delayed stochastic signals $x(t)$ and $y(t)[20$, $21,23,26]$. An exemplary time records of the signals (after centering and filtration) obtained for the air-water flow in the experiment denoted as "BUB010" are presented in figure 3 .

\section{The analysis of signals}

The analyses of signals from scintillation probes allow determining of the transportation time delay $\tau_{0}$ and then the mean velocity $v_{A}$ of gas phase transportation, from the following formula:

$$
v_{A}=L / \tau_{0}
$$

The most known classical methods of time delay estimation of an ergodic random signals is based on the cross - correlation function, defined as follow [13]:

$$
R_{x y}(\tau)=\lim _{T \rightarrow \infty} \frac{1}{T} \int_{0}^{T} x(t) y(t+\tau) d t,
$$


where $T$ is the averaging time, $\tau$ - time delay.
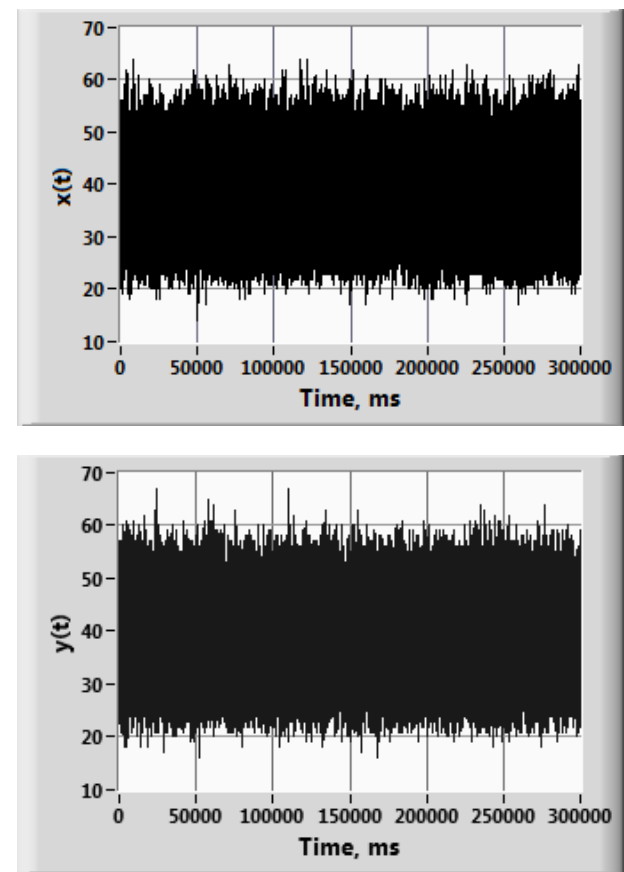

Figure 3. Time records of signal $x(t)$ and $y(t)$ (after preprocessing) obtained in the BUB010 experiment.

Following normalisation, the correlation (4) is equal to:

$$
\rho_{x y}(\tau)=\frac{R_{x y}(\tau)}{\sigma_{x} \cdot \sigma_{y}}
$$

where $\sigma_{x}$ and $\sigma_{y}$ are standard deviations of $x(t)$ and $y(t)$ signals respectively.

The $\tau_{0}$ transportation time delay is determined on the base of position of the main maximum of the CCF. The discrete estimator for the cross-correlation function can be expressed by the following formula:

$$
\hat{R}_{x y}(\tau)=\frac{1}{N} \sum_{n=0}^{N-1} x(n) \cdot y(n+\tau)
$$

where $N$ is the number of values of discrete $x(n)$ and $y(n)$ signals, and $n=t / \Delta t[28-30]$.

In papers [22 - 25] for analysis of stochastic signals the conditional averaging has been proposed. In this technique for the time delay determination the principle of the minimum of the conditional expected value was used. This value of the delayed signal $y(t)$ for $x(t)=0$ can be presented by the relationship:

$$
A_{|y|}=A_{|y| \mid x=0}=E\left\{\left.|y|\right|_{x=0}\right\}=\int_{0}^{\infty}|y| \cdot p\left(\left.|y|\right|_{x=0}\right) d y,
$$

where for the simplifying of the notation it was assumed that $y(t)=y, x(t)=x$, and $p\left(\mid y \|_{x=0}\right)$ is the conditional probability of the density function of the signal absolute value $y$ for $x=0$ [22].

The normalized $a_{|y|}(\tau)$ function is defined as:

$$
a_{|y|}(\tau)=\frac{A_{|y|}(\tau)}{A_{|y|}(\tau)_{\max }}
$$

A good estimator of the conditional expected value (7) is CAAV. In practice it is determined by the detection of the signal $x$ passes through zero and then recording the signals $y$ and averaging the absolute values of them. The discrete estimator CAAV can be written in the following form:

$$
\hat{A}_{|y|}(\tau)=\left.\overline{\mid y(n+\tau)}\right|_{x(n)=0}=\left.\frac{1}{M} \sum_{n=1}^{M}|y(n+\tau)|\right|_{x(n)=0},
$$

where $M$ is the number of passes through the zero of the signal $x(n)$.

In the case of CAAV the determination of $\tau_{0}$ is based on finding the main minimum of this function.

In this work we propose to use the combined $\mathrm{CCF} / \mathrm{CAAV}$ calculation for analysis of signals obtained in gamma-absorption measurements of the liquid-gas mixture transportation. This calculation is as follows:

$$
R_{C C F / C A A V}(\tau)=\frac{R_{x y}(\tau)}{A_{|y|}(\tau)},
$$

or for normalized CCF and CAAV functions:

$$
R_{C C F / C A A V}(\tau)=\frac{\rho_{x y}(\tau)}{a_{|y|}(\tau)} .
$$

In theoretical considerations of the noise-free signals a small positive numbers should be added to the denominators of the equations (10) and (11) to avoid division by zero. However, for the analysis of real signals this is not necessary.

\section{Exemplary results}

Figure 4a presents plots of the normalized CCF (5) and $\mathrm{CCF} / \mathrm{CAAV}$ (11) characteristics obtained for signals recorded in the BUB010 experiment. In figure $4 \mathrm{~b}$ additional normalization of each function by its maximum value was done. In the BUB010 experiment, the laminar flow was analyzed and the bandwidth of signals was about $20 \mathrm{~Hz}$. The data acquisition parameters in this experiment were: $N=300000, \Delta t=1 \mathrm{~ms}$. In both methods, the filtration of recorded signals was used in order to noises reduction.

In calculations of transportation time delay the selected range of data around the maximum of the CCF and $\mathrm{CCF} / \mathrm{CAAV}$ have been interpolated with the Gaussian probability density distribution $p(\tau)$ :

$$
p(\tau)=p_{0}+\frac{1}{\sigma \cdot \sqrt{2 \pi}} \exp \left(-\frac{\left(\tau-\hat{\tau}_{0}\right)^{2}}{2 \cdot \sigma^{2}}\right),
$$

where: $p_{0}-$ normalization level of the Gauss function, $\sigma-$ standard deviation of the fitted distribution. 
a)

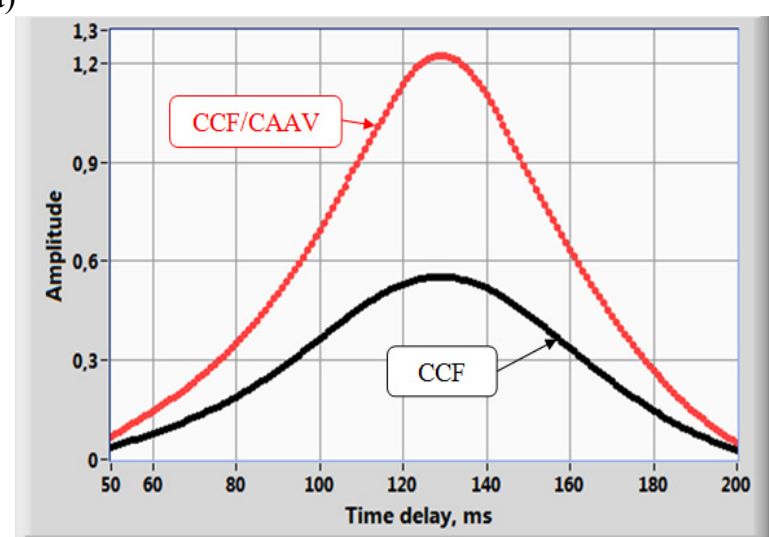

b)

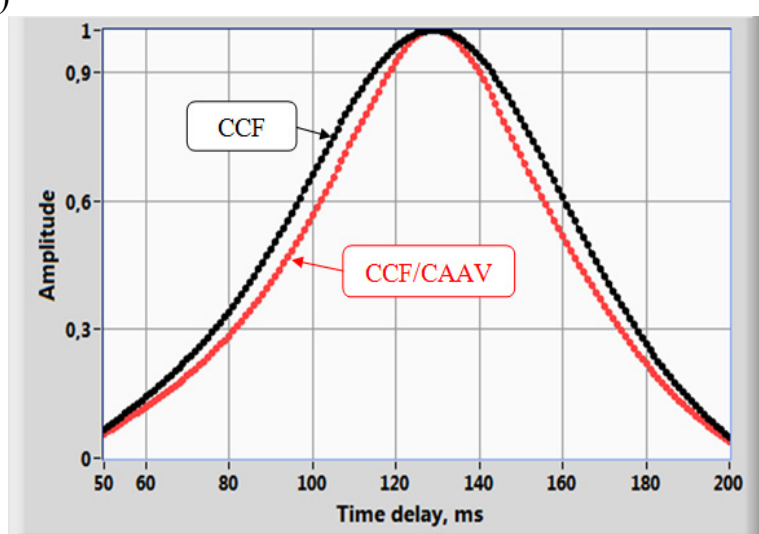

Figure 4. (a) Comparison of the normalized $\mathrm{CCF}$ and $\mathrm{CCF} / \mathrm{CAAV}$ functions calculated in the BUB010 experiment, (b) the same functions after additional amplitude normalization.

Therefore the $\hat{\tau}_{0}$ estimator of the mean transportation time is determined as the first moment of the fitted distributions [10, 20,27] and the standard uncertainty $u\left(\hat{\tau}_{0}\right)$ is given by:

$$
u\left(\hat{\tau}_{0}\right)=\sigma / \sqrt{q},
$$

where $q-$ number of points selected for interpolation.

In table 1 results of the time delay estimation and its standard uncertainty obtained in the BUB010 experiment for $q=126$ are presented.

Table 1. Time delay estimation in the BUB010 experiment.

\begin{tabular}{|c|c|c|}
\hline Method & $\begin{array}{c}\text { Time delay } \\
\hat{\tau}_{0}, \mathrm{~ms}\end{array}$ & $\begin{array}{c}\text { Standard } \\
\text { uncertainty } \\
u\left(\hat{\tau}_{0}\right), \mathrm{ms}\end{array}$ \\
\hline $\mathrm{CCF}$ & 128.45 & 2.65 \\
\hline $\mathrm{CCF} / \mathrm{CAAV}$ & 128.30 & 2.27 \\
\hline
\end{tabular}

The combined standard uncertainty $u_{c}\left(v_{A}\right)$ of the gas phase velocity, with negligible small uncertainties of the acquisition set, depends on an inaccuracy of uncorrelated $L$ and $\hat{\tau}_{0}$ values determination:

$$
u_{c}\left(v_{A}\right)=\left[\left(\frac{\partial v_{A}}{\partial L}\right)^{2} u^{2}(L)+\left(\frac{\partial v_{A}}{\partial \hat{\tau}_{0}}\right)^{2} u^{2}\left(\hat{\tau}_{0}\right)\right]^{1 / 2},
$$

where $u(L)$ is the standard uncertainty of the distance between the detectors.

Results of the mean velocity $v_{A}$ and its combined uncertainty $u_{c}\left(v_{A}\right)$ [31] obtained in the BUB010 experiment are presented in the table 2.

Table 2. Mean velocity of air $v_{A}$ and its uncertainty $u_{c}\left(v_{A}\right)$.

\begin{tabular}{|c|c|c|}
\hline Method & $\begin{array}{c}\text { Mean velocity } \\
v_{A}, \mathrm{~m} / \mathrm{s}\end{array}$ & $\begin{array}{c}\text { Combined } \\
\text { uncertainty } \\
u_{c}\left(v_{A}\right), \mathrm{m} / \mathrm{s}\end{array}$ \\
\hline $\mathrm{CCF}$ & 0.755 & 0.016 \\
\hline $\mathrm{CCF} / \mathrm{CAAV}$ & 0.756 & 0.013 \\
\hline
\end{tabular}

\section{Conclusions}

Based on the preliminary results it can be stated that the $\mathrm{CCF} / \mathrm{CAAV}$ method can be used in gamma-absorption measurements for mean gas bubbles velocity determination of the liquid-gas mixture flow in the horizontal pipeline. The results of the time delay and air bubbles velocity obtained in the BUB010 experiment by $\mathrm{CCF} / \mathrm{CAAV}$ and $\mathrm{CCF}$ methods were similar. The standard uncertainty of time delay measurement using $\mathrm{CCF} / \mathrm{CAAV}$ gives $8.6 \%$ lower value than one delivered by the CCF alone. The combined uncertainties of the mean velocity of air bubbles evaluation in the presented experiment did not exceed $2.1 \%$ (CCF) and $1.7 \%$ (CAAV), which are satisfactory results in industrial applications.

\section{References}

1. G. Falcone, G.F. Hewitt, C. Alimonti, Multiphase flow metering: principles and applications (Elsevier Amsterdam, 2009)

2. M. Bottin, et al, Int. J. Multiphas. Flow 60, 161-179 (2014)

3. T. Xue, L. Qu, Z. Cao, T. Zhang, Flow Meas. Instrum. 27, 29-36 (2012)

4. M.R. Rzasa, Nucl. Eng. Des. 239, 699-707 (2009)

5. G.A. Johansen, P. Jackson, Radioisotope gauges for industrial process measurements (John Wiley, New York 2004)

6. Z. Yu, B. Qincheng, H. Richa, Appl. Therm. Eng. 60, 398-410 (2013)

7. W.A.S. Kumara, B.M. Halvorsen, M.C. Melaaen, Int. J. Multiphas. Flow 36, 467-480 (2010)

8. B.K. Arvoh, R. Hoffmann, M. Halstensen, Flow Meas. Instrum. 23, 56-65 (2012)

9. S.H. Jung, et al, Appl. Rad. Isotop. 67, 1254-1258 (2009) 
10. M. Zych, et al, Flow Meas. Instrum. 35, 11-15 (2014)

11. L. Petryka, et al, Prz. Elektrotech. 88 (1b), 185-188 (2012)

12. E. Pawlowski, Proc. SPIE 4516, 181-186 (2001)

13. J.S. Bendat, A.G. Piersol, Random data - analysis and measurement procedures (John Wiley, New York, 2010)

14. V. Mosorov, Meas. Sci. Technol. 17, 753-760 (2006)

15. R. Hanus, Prz. Elektrotech. 84 (12), 301-303 (2008)

16. A.G. Piersol, IEEE Trans. Acoust. Speech Sign Proc. 29, 471-477 (1981)

17. J.S. Bendat, The Hilbert Transform and applications to correlation measurements Brüel\&Kjær BT000811 (Naerum, Denmark, 1985)

18. R. Hanus, Prz. Elektrotech. 88 (10b), 39-41 (2012)

19. G. Jacovitti, G. Scarano, IEEE Trans. Sign. Proc 41, 525-533 (1993)

20. R. Hanus, L. Petryka, M. Zych, Flow Meas. Instrum. 40, 58-63 (2014)

21. R. Hanus, M. Zych, L. Petryka, JPCS 530012042 (2014)
22. A. Kowalczyk et al, Metrol. Meas. Syst. 18, 335342 (2011)

23. R. Hanus, et al, Proc. 2012 IEEE Mediterranean Electrotechnical Conference MELECON 2012, 144147 (2012)

24. A. Kowalczyk, A. Szlachta, Prz. Elektrotech. 86 (1), 225-228 (2010)

25. R. Hanus, Prz. Elektrotech. 86 (6), 232-235 (2010)

26. D. Swisulski, Prz. Elektrotech. 88 (10b), 29-31 (2012)

27. L. Petryka, et al, Proc. 10th ISOPE Ocean Mining \& Gas Hydrates Symp. OMS-2013, 230-233 (2013)

28. M.S. Beck, A. Plaskowski, Cross correlation flowmeters - their design and application (Adam Hilger, Bristol, 1987)

29. W.Q. Yang, M.S. Beck, Flow Meas. Instrum. 8, 7784 (1998)

30. S.L. Soo (ed.), Instrumentation for fluid-particle flow (Noyes Publications, New Jersey, 1999)

31. Guide to the expression of uncertainly in measurement (International Organisation for Standardisation, 1995) 